\title{
Assessing the utility of CASP14 models for molecular replacement
}

\author{
Claudia Millán ${ }^{1}$, Ronan Keegan ${ }^{2}$, Joana Pereira ${ }^{3}$, Massimo Domenico Sammito ${ }^{1}$, Adam \\ Simpkin ${ }^{4}$, Airlie McCoy ${ }^{1}$, Andrei N. Lupas ${ }^{5}$, Marcus Hartmann ${ }^{6}$, Daniel Rigden ${ }^{4}$, and \\ Randy Read ${ }^{1}$ \\ ${ }^{1}$ University of Cambridge \\ ${ }^{2}$ UKRI Science and Technology Facilities Council \\ ${ }^{3}$ Universitat Basel \\ ${ }^{4}$ University of Liverpool \\ ${ }^{5}$ Max-Planck-Inst. for Developmental Biology \\ ${ }^{6}$ Max Planck Institute for Developmental Biology
}

June 19, 2021

\begin{abstract}
The assessment of CASP models for utility in molecular replacement is a measure of their use in a valuable real-world application. In CASP7, the metric for molecular replacement assessment involved full likelihood-based molecular replacement searches; however, this restricted the assessable targets to crystal structures with only one copy of the target in the asymmetric unit, and to those where the search found the correct pose. In CASP10, full molecular replacement searches were replaced by likelihoodbased rigid-body refinement of models superimposed on the target using the LGA algorithm, with the metric being the refined likelihood (LLG) score. This enabled multi-copy targets and very poor models to be evaluated, but a significant further issue remained: the requirement of diffraction data for assessment. We introduce here the relative-expected-LLG (reLLG), which is independent of diffraction data. This reLLG is also independent of any crystal form, and can be calculated regardless of the source of the target, be it X-ray, NMR or cryo-EM. We calibrate the reLLG against the LLG for targets in CASP14, showing that it is a robust measure of both model and group ranking. Like the LLG, the reLLG shows that accurate coordinate error estimates add substantial value to predicted models. We find that refinement by CASP groups can often convert an inadequate initial model into a successful MR search model. Consistent with findings from others, we show that the AlphaFold2 models are sufficiently good, and reliably so, to surpass other current model generation strategies for attempting molecular replacement phasing.
\end{abstract}

\section{Hosted file}

CASP14_MR_submitted_flat.docx available at https://authorea.com/users/420606/articles/526917assessing-the-utility-of-casp14-models-for-molecular-replacement 